\title{
Anisotropic viscous dissipation in compressible magnetic $X$-points
}

\author{
I. J. D. Craig ${ }^{1}$
}

Department of Mathematics, University of Waikato, PB 3105, Hamilton, New Zealand

Received 14 April 2008 / Accepted 20 June 2008

\begin{abstract}
Context. Visco-resistive damping in line-tied magnetic $X$-points is examined.

Aims. The goal is to determine whether fast, Alfvénic energy dissipation is possible for $X$-point disturbances damped by the plasma resistivity and non-isotropic viscosity.

Methods. The response of $X$-points to planar and axial perturbations is explored numerically by solving the linearized compressible MHD equations in two and a half dimensions.

Results. It is demonstrated that fast dissipation is possible in the case of non-reconnective planar disturbances damped by anisotropic viscosity in weakly resistive plasmas. Although perturbations which change the initial $X$-point topology decay slowly at large times when viscous effects are dominant, there is an initial phase in which a significant fraction of the disturbance energy is removed on an Alfvénic timescale. The decay of incompressive axial field disturbances occurs by a different mechanism, however, that is always formally slow (i.e. dependent on the small viscous and resistive damping coefficients).

Conclusions. Computations suggest that fast, visco-resistive energy release in coronal plasmas is possible for compressive $X$-point disturbances. This result could have important implications for understanding rapid energy release in coronal active regions.
\end{abstract}

Key words. magnetohydrodynamics (MHD) - Sun: flares - Sun: magnetic fields - Sun: corona - plasmas

\section{Introduction}

A recurring difficulty in coronal physics is explaining the explosive energy release observed in solar flares. Although magnetic reconnection is the favoured mechanism of energy release, the weak coronal resistivity leads to energy dissipation rates that are generally too slow to account for the explosive phase of the flare (Priest \& Forbes 2000). Accordingly, recent theoretical work has focused on accelerating the energy losses by incorporating other physical ingredients into the reconnection mechanism. Non-collisional effects, for example, have been explored by adopting a generalized form of Ohm's law that accounts for Hall currents and finite electron inertia (e.g. Knoll \& Chacon 2006). The Hall MHD equations may well provide the simplest physical model capable of describing fast collisionless reconnection (Birn et al. 2001).

Another possibility for modifying the energy release rate is to incorporate viscous damping into the reconnection model (Craig et al. 2005). Some observational support for the presence of viscous effects in coronal plasmas can be inferred from flare outflow speeds: these appear considerably lower than the Alfvénic exhausts predicted on standard resistive models (Asai et al. 2004). More generally, since dimensionless viscosity coefficients can exceed the normalized coronal resistivity by several orders of magnitude, viscous effects seem a natural candidate for inclusion in coronal energy release models (Hollweg 1985, 1986). The matter seems less clear cut, however, when it is remembered that viscous damping of the velocity field is incapable of removing energy by simplifying the magnetic field topology - only reconnection acting through the plasma resistivity can achieve this aim. A further complication is that the viscosity is highly non-isotropic in plasmas with strong magnetic fields (Braginskii 1965). Therefore visco-resistive computations in coronal plasmas are likely to be very sensitive to the field geometry.

The purpose of this paper is to investigate how anisotropic viscosity affects magnetic energy release in a line-tied $X$-point geometry. This problem provides one of the simplest geometries available to us and allows a side by side comparison between isotropic viscosity models and more exact anisotropic treatments. Of particular interest is the extent to which anisotropic viscosity can maintain strong energy damping, both for shearing disturbances normal to the plane of $X$-point, and for compressive planar disturbances. The present analysis extends the work of Craig Senanayake \& Litvinenko (2005) who considered only isotropic viscous damping of compressive $X$-point disturbances; it also complements Craig \& Litvinenko (2007) who examined finite amplitude axial field dissipation in the case of anisotropic viscosity.

In Sect. 2 we formulate the $X$-point problem in the case of visco-resistive damping for both traditional and non-isotropic viscosities. The analysis is based on linearized MHD equations in a two and one half dimensional, line-tied geometry. Our central results are presented in Sect. 3 where we compute the global energy losses of the system using a variety of initial conditions. In Sect. 4 we summarize our findings.

\section{Energy dissipation in magnetic $X$-points}

\subsection{Introduction}

The problem we consider is the evolution of a line-tied, magnetic $X$-point. The $X$-point is disturbed by some global, initial perturbation and relaxes by visco-resistive damping, to a current free equilibrium. Our main concern is the effectiveness of the damping mechanisms. Can the energy be released rapidly on an 
Alfvénic timescale, or is the release rate inhibited by the small viscous and resistive damping coefficients?

As in previous treatments (Craig et al. 2005), we work with the compressible MHD equations, scaled with respect to typical coronal values for field strength $B_{\mathrm{c}}=10^{2} \mathrm{G}$, size scale $l_{\mathrm{c}}=$ $10^{9.5} \mathrm{~cm}$ and number density $n_{\mathrm{c}}=10^{9} \mathrm{~cm}^{-3}$. Times are measured in units of $l_{\mathrm{c}} / v_{\mathrm{A}}$ where $v_{\mathrm{A}}=10^{9} \mathrm{~cm} \mathrm{~s}^{-1}$ is the Alfvén speed.

Energy losses from the source volume are controlled by two small parameters, namely, the dimensionless resistivity $\eta$ and the dimensionless plasma viscosity $v$. Note that $\eta$ is an inverse Lundquist number

$\eta=\frac{c^{2}}{4 \pi v_{\mathrm{A}} l_{\mathrm{c}} \sigma} \simeq 10^{-14.5}$

based on collisional plasma of $T=10^{6} \mathrm{~K}$ with conductivity $\sigma \sim T^{3 / 2}$ (Spitzer 1962). The viscosity coefficient

$v=\frac{\bar{v}}{\rho_{\mathrm{c}} v_{\mathrm{A}} l_{\mathrm{c}}} \simeq 10^{-4.5}$,

derives from a plasma of mass density $\rho_{\mathrm{c}}$ in which viscosity scales as $\bar{v} \sim T^{5 / 2}$.

Given that $v \gg \eta$ it is natural to assume that viscous losses generally dominate resistive losses. This is especially likely in hot active region plasmas where viscous effects may be enhanced by temperatures of several million degrees and above. However, if the initial disturbance involves changes in the plasma-field topology, the energy release cannot be independent of the plasma resistivity. Magnetic reconnection is then required to restore the $X$-point to its initial state and viscosity, acting alone, can damp only those parts of the disturbance that manifest themselves in terms of the global kinetic energy.

\subsection{MHD equations and boundary conditions}

We consider the case of small displacements about a cold, two and a half dimensional $X$-point plasma with $\partial_{z}=0$. The plasma is of uniform density and confined to the region $|x|,|y|<1$. The boundary $\Omega$ is idealized as a highly conducting rigid surface that anchors the magnetic field lines.

We use the flux function representation

$$
\boldsymbol{B}(x, y, t)=\nabla \psi \times \hat{z}+Z \hat{z}
$$

and split the magnetic and velocity fields into planar and axial components

$$
\boldsymbol{B}(x, y, t)=\left(\partial_{y} \psi,-\partial_{x} \psi, Z\right), \quad \boldsymbol{v}(x, y, t)=(\boldsymbol{u}, W) .
$$

The evolution equations are obtained by linearizing about a static planar, background field $\boldsymbol{B}_{E}$. If we let

$$
\psi(x, y, t) \rightarrow \psi_{E}(x, y)+\psi(x, y, t)
$$

and write $\boldsymbol{F}$ for the viscous forces then the planar and axial field perturbations satisfy

$$
\begin{aligned}
& \frac{\partial \boldsymbol{u}}{\partial t}=-\nabla^{2} \psi \nabla \psi_{E}+\boldsymbol{F}_{\perp} \\
& \frac{\partial \psi}{\partial t}+\boldsymbol{u} \cdot \nabla \psi_{E}=\eta \nabla^{2} \psi, \\
& \frac{\partial W}{\partial t}=\left(\boldsymbol{B}_{E} \cdot \nabla\right) Z+F_{\|}, \\
& \frac{\partial Z}{\partial t}=\left(\boldsymbol{B}_{E} \cdot \nabla\right) W+\eta \nabla^{2} Z .
\end{aligned}
$$

(9) $S=\frac{2}{3} \partial_{x} U-\frac{1}{3}\left(\partial_{y} V+\partial_{z} W\right)$
Viscous forces are computed by specifying a suitable stress tensor $\mathcal{S}$ for the plasma

$\boldsymbol{F}=\left(\boldsymbol{F}_{\perp}, F_{\|}\right)=\nabla \cdot \mathcal{S}$,

as discussed below. For the moment we note that in the absence of viscous damping it is possible for the planar and axial fields to evolve independently. Thus if only the axial fields are initially disturbed we can take $\psi$ and $\boldsymbol{u}$ to vanish for all time. This decoupling is no longer assured when anisotropies in the viscosity are accounted for.

In accordance with the assumption of line-tying, $\psi$ is fixed on the boundary $\Omega$ where both the velocity and the normal gradient of $Z$ are taken to vanish:

$\partial_{t} \psi=\partial_{n} Z=|\boldsymbol{v}|=0 \quad$ on $\Omega$.

These conditions ensure that energy losses occur only from viscous and resistive dissipation within the source volume. As shown in Sect. 2.4, no energy is transported through the boundary of the $X$-point.

\subsection{Form of the viscous stress tensor}

Turning now to the form of the plasma viscosity, we note that the classical hydrodynamic expression derives from the tensor

$\mathcal{S}_{i j}=v\left(\partial_{j} v_{i}+\partial_{i} v_{j}-\frac{2}{3} \delta_{i j} \nabla \cdot v\right)$

This form is no longer accurate for a magnetized collisional plasma in which the proton mean free path greatly exceeds the proton gyro-radius. In such cases the cross field viscosity is greatly inhibited and the strong field Braginskii (1965) form is appropriate

$\mathcal{S}_{i j}=v_{0}\left(3 \frac{B_{i} B_{j}}{B^{2}}-\delta_{i j}\right)\left(\frac{B_{m} B_{k}}{B^{2}} \partial_{k} v_{m}-\frac{1}{3} \nabla \cdot v\right)$,

where $v_{0} \simeq v$ (Hollweg 1985) and summation over repeated suffixes is assumed. This expression is fully warranted for the damping of coronal $X$-point plasmas where field strengths of order $10^{2}$ Gauss are expected. The only possible exception is provided by the weak field region at the origin of the $X$-point which - because the proton gyro-radius approaches physically significant scales only for fields of $10^{-4} \mathrm{G}$ or smaller - is a region of almost negligible measure.

To highlight the role of the strong field viscosity, note that the classical viscosity provides a force

$\boldsymbol{F}=v\left[\nabla^{2} \boldsymbol{v}+\frac{1}{3} \nabla(\nabla \cdot \boldsymbol{v})\right]$

that is independent of the magnetic field. The only term with this property in the Braginskii expression derives from the finite compressibility of the plasma $(\nabla \cdot v)$. It follows that the damping of incompressible disturbances is very sensitive to the geometry of the background field (Craig \& Litvinenko 2007).

We conclude that expression (13) may lead to forces that depend in a complicated way on the details of the magnetic geometry. But one case which can be easily illustrated is that of a one-component field, say $\boldsymbol{B}=\left(B_{1}, 0,0\right)$, advected by a threedimensional flow $\boldsymbol{v}=(U, V, W)$. The second bracket in (13) yields the scalar 
and the force becomes

$\boldsymbol{F}=v\left(\begin{array}{ccc}2 & 0 & 0 \\ 0 & -1 & 0 \\ 0 & 0 & -1\end{array}\right)\left(\begin{array}{l}\partial_{x} S \\ \partial_{y} S \\ \partial_{z} S\end{array}\right)$.

The special status of the first component of the force is a reflection of the anisotropy of the underlying $\boldsymbol{B}$-field. Note that, although this expression appears independent of the field strength, it cannot be extrapolated to weak fields because of the strong field approximation inherent in the derivation of (13).

For completeness it should also be mentioned that in a magnetically stratified, collisional plasma the parallel and perpendicular components of the resistivity can vary by a factor of around two. Since we generally assume $v \gg \eta$ this effect is neglected in the present treatment.

\subsection{Global energy losses}

Finally, we obtain a general expression for the global energy losses in our system valid for all forms of viscosity. We first dot equation (6) with $\boldsymbol{u}$, and substitute for $\boldsymbol{u} \cdot \nabla \psi_{E}$ from (7). Integrating over the volume and using (11) to eliminate surface contributions we find that

$\frac{\mathrm{d}}{\mathrm{d} t} \int \frac{1}{2}\left(u^{2}+(\nabla \psi)^{2}\right) \mathrm{d} V=\int\left(\boldsymbol{u} \cdot \boldsymbol{F}_{\perp}-\eta\left(\nabla^{2} \psi\right)^{2}\right) \mathrm{d} V$.

The losses for the axial components are calculated in a similar way:

$\frac{\mathrm{d}}{\mathrm{d} t} \int \frac{1}{2}\left(W^{2}+Z^{2}\right) \mathrm{d} V=\int\left(W F_{\|}-\eta(\nabla Z)^{2}\right) \mathrm{d} V$.

By adding (18) to (17) and writing the perturbation fields in the form

$\boldsymbol{b}=\left(\partial_{y} \psi,-\partial_{x} \psi, Z\right), \quad \boldsymbol{J}=\left(Z_{y},-Z_{x},-\nabla^{2} \psi\right)$,

we obtain a physically transparent form for the global energy losses, namely

$\dot{\mathcal{E}} \equiv \frac{\mathrm{d}}{\mathrm{d} t} \int \frac{1}{2}\left(b^{2}+v^{2}\right) \mathrm{d} V=\int\left(\boldsymbol{v} \cdot \boldsymbol{F}-\eta J^{2}\right) \mathrm{d} V$.

This form clearly distinguishes the work done by viscous forces against the Ohmic losses $\eta J^{2}$ of the plasma.

In considering expression (20) we should remember that equipartition between the kinetic and magnetic energies is expected only for ideal plasmas. This follows from the symmetry of the ideal, incompressible MHD equations under the Elsässer variables $\boldsymbol{b} \pm \boldsymbol{v}$. More generally, if both resistivity and viscosity contribute to the damping, then the kinetic and magnetic energies are liable to separate over long times, especially for disparate resistive and viscous damping coefficients. In fact this separation is guaranteed for disturbances that alter the initial $X$ point topology yet are damped predominantly by viscosity: the kinetic energy may dissipate rapidly but a slowly decaying residual of topological magnetic energy will remain.

\section{Viscous dissipation of $X$-point disturbances}

\subsection{Theory of $X$-point damping}

The computations that follow are based on the current free equilibrium field $\psi_{E}=x y$ over the domain $-1 \leq x, y \leq 1$. Although any combination of planar and axial field disturbances can be used to perturb the $X$-point, it is convenient to specify initial conditions according to:

(1) planar disturbances that maintain the initial $X$-point topology;

(2) planar disturbances that change the $X$-point topology;

(3) pure axial field disturbances.

The first two classes have already been discussed in the context of isotropic viscosity (Craig et al. 2005) and our aim is to to extend this treatment to the more realistic Braginskii form (13). We shall concentrate mainly on the energetics of viscously dominated plasmas and neglect the detailed structure of the disturbance fields. But no matter which viscosity is modelled, perturbations of type (1) and (2) drive compressive modes without inducing axial field disturbances. Thus there is no mixing of compressive and shearing disturbances.and we can take $Z=W=0$ for all time.

Disturbances of type (3) are quite different: they initially drive non-compressive shear waves perpendicular to the plane of the $X$-point so $\psi=u=0$. However, when the Braginskii tensor (13) is adopted, viscous forces in the plane are eventually generated. This coupling provides an avenue for energy transfer between the axial and planar fields that is not present in the isotropic viscosity model.

Turning now to the theory of $X$-point plasmas, we note that attention has mainly focused on planar resistive dissipation with small or negligible viscosity $(\eta \gg v)$. Since resistivity allows field line reconnections to occur, no distinction need be made between disturbances of types (1) and (2). Specifically, the resistive system can be understood as an underdamped oscillator (Craig \& McClymont 1991, 1993; Hassam 1992) whose decay is governed by the fundamental cylindrical eigenmode $\psi \sim \exp (-\lambda t)$ where

$\lambda=\alpha+\mathrm{i} \omega, \quad \omega=\frac{\pi}{|\ln \eta|}, \quad \alpha=\frac{1}{2} \omega^{2}$.

The oscillation frequency is determined by the time it takes an Alfvén wave launched from the outer boundary $r=r_{\mathrm{b}} \simeq 1$ to impact the localized diffusion region of size $r_{s}$. Since $v_{\mathrm{A}}=r$ in our units we obtain the "bounce time"

$\tau_{\mathrm{B}}=\int_{r_{s}}^{r_{\mathrm{b}}} \frac{\mathrm{d} r}{r} \simeq \frac{1}{2}|\ln \eta|$

on setting $r_{s} \simeq \sqrt{\eta}$. The fundamental mode emerges after an initial transient phase, typically lasting a few Alfvén times, and leads to "fast" damping of the global energy (with rate $2 \alpha$ ). At very long times the fundamental mode gives way to a localized self-similar mode, but the perturbation energy is insignificant by this stage. In practice, it is known that fast reconnection can be stalled by back pressures that build up and halt the collapse to the resistive scale $\sqrt{\eta}$ (Deluca \& Craig 2002; McClymont \& Craig 1996). This effect depends on the plasma beta and is neglected in the present (cold plasma) limit.

Disturbances of type (3) are damped by a completely different mechanism. Initially there is a quasi-ideal phase of phase mixing (Heyverts \& Priest 1983) in which axial field corrugations build up along the planar field lines. These corrugations grow until field gradients are steep enough to be dissipated either by viscosity or resistivity (Fruit \& Craig 2006). For $v \gg \eta$ isotropic viscosity is known to limit the severity of the shear and provide significant damping on the - formally slow - timescale $v^{-1 / 3}$ However, as the strong field Braginskii form (13) effectively suppresses the shear viscosity, it is expected to be significantly less effective as an energy loss mechanism. 


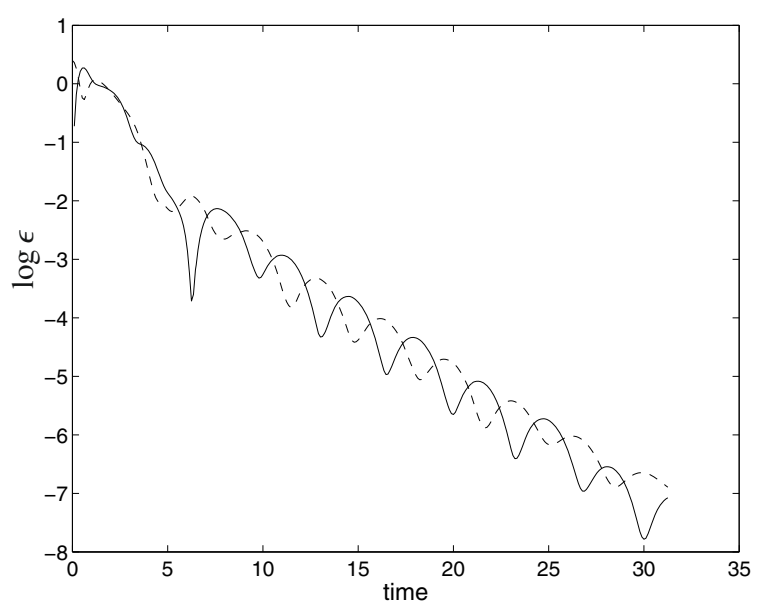

Fig. 1. Plot of the $\log _{10}$ kinetic and magnetic (solid line) energies against time for a non-reconnective disturbance. Parameters are $v=$ $2 \times 10^{-3}, \eta=2 \times 10^{-4}$ and isotropic viscosity is assumed.

\subsection{Non-reconnective planar disturbances}

We turn now to disturbances of type (1) and use

$$
\begin{aligned}
\psi(x, y, 0) & =\left(1-x^{2}\right)\left(1-y^{2}\right) \exp \left(-s^{2}\right), \\
s & =\frac{1}{2} \ln \left(x^{2}+y^{2}\right)
\end{aligned}
$$

with $v=0$ as a typical initial condition (Craig et al. 2005). In typical runs we set $\eta=0.1 v$. Figures 1 and 2 show the decay of the global $X$-point energy for isotropic and anisotropic viscosity models. These computations assume $v=2 \times 10^{-3}$ but the decay profiles are typical of runs in the range $10^{-4} \leq v \leq 10^{-2}$ with $\eta<v$.

Consider first Fig. 1, which summarizes the decay of the magnetic and kinetic energies in the case of an isotropic viscosity. In line with previous work, the decay is found to exhibit three well-defined phases: an initial rapid decline in the global energy $(0 \leq t \leq 10)$; a stage of fast oscillatory decay in which the magnetic and kinetic energies continually interchange $(10 \leq t \leq 30)$; finally, an asymptotic phase which involves the slow, non-oscillatory separation of the global magnetic and kinetic energies $t \geq 30$ ).

The first two stages are well represented in Fig. 1 but the final phase is only just starting to emerge. However, if we focus on the effectiveness of viscous dissipation, the final decay is largely irrelevant since all but a small residual of the global energy has been dissipated. In particular, a peak damping rate of order unity is achieved during the initial stages $(t \simeq 2.5)$ and the losses remain substantial throughout the subsequent oscillatory phase. These rates are found to depend at most logarithmically on the viscous coefficient $v$. Notably, the phase of oscillatory damping (in which $\mathcal{E} \sim \exp (-\gamma t)$ with $\gamma \simeq 0.45$ ) appears to provide a viscous analogue of the fundamental resistive mode described by Eq. (21). In fact our computations indicate that the viscous damping rate $(\eta=0)$ during the oscillatory phase is slightly faster than the pure resistive damping rate according to $\alpha_{v} \simeq 1.4 \alpha$.

Figure 2 shows the corresponding computation based on the anisotropic viscosity. The separate phases of Fig. 1 are still present but they are no longer so well defined. There is also a tendency for the magnetic and kinetic energies to separate more readily at larger times. Despite these differences, the decay rates in the first and second phases remain strong, comparable in fact

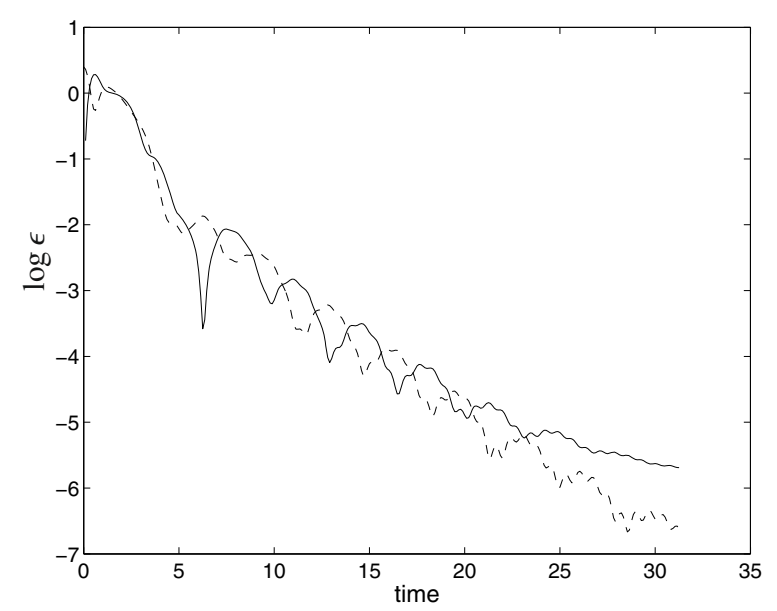

Fig. 2. Same as Fig. 1 except that anisotropic viscosity is assumed $(v=$ $\left.2 \times 10^{-3}, \eta=2 \times 10^{-4}\right)$. Solid line indicates global magnetic energy

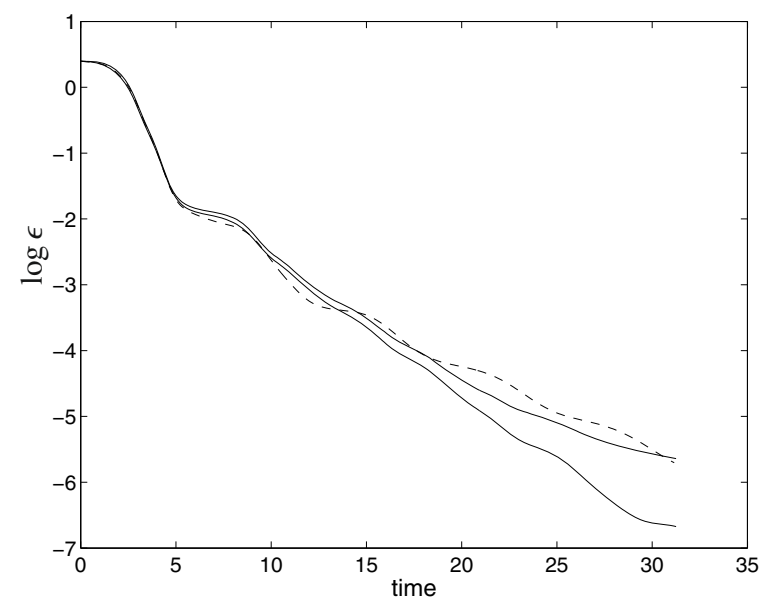

Fig. 3. Solid line shows plots of the total disturbance energy versus time based on the computations of Figs. 1 and 2. The lower curve (fastest decay) is obtained for the isotropic viscosity but the Braginskii viscosity is only marginally slower. The dotted line shows a run in which resistivity dominates for the case $\eta=2 \times 10^{-3}, v=2 \times 10^{-4}$.

to those of the isotropic viscous computation. Since this behaviour holds good for all viscosity's in the physically plausible range $10^{-4} \leq v \leq 10^{-2}$, it is natural to assume that the compressible components in the viscous stress tensor (13) are primarily responsible for the global energy losses (see also Fig. 8 below). This interpretation is reinforced by Fig. 3 which provides a side by side comparison of the global energy decay $\dot{\mathcal{E}}$ for the two viscous models. Also shown (dotted lines) is the damping profile for the case in which resistivity is dominant $\left(\eta=2 \times 10^{-3}, v=0.1 \eta\right.$, isotropic viscosity being assumed). As far as the initial energy losses are concerned, all three models provide equally effective damping. Minor variations do occur but these become significant only in the later phases by which time $(t>20)$ the disturbance energy has been reduced by factors of order $10^{-5}$.

Finally, in Fig. 4 we show scalings for the peak energy loss rate $(\max (\dot{\mathcal{E}}))$ together with the time $T$ at which the peak rate is achieved. This rate is the outcome of an initial implosion that drives the "first bounce" of the disturbance off the origin (22). Notably, the Braginskii viscosity achieves a marginally faster rate at the cost of a slightly longer bounce time (that still only lasts a few Alfvén times). The energy loss rate is almost independent of the viscous coefficient for both models, but there is 


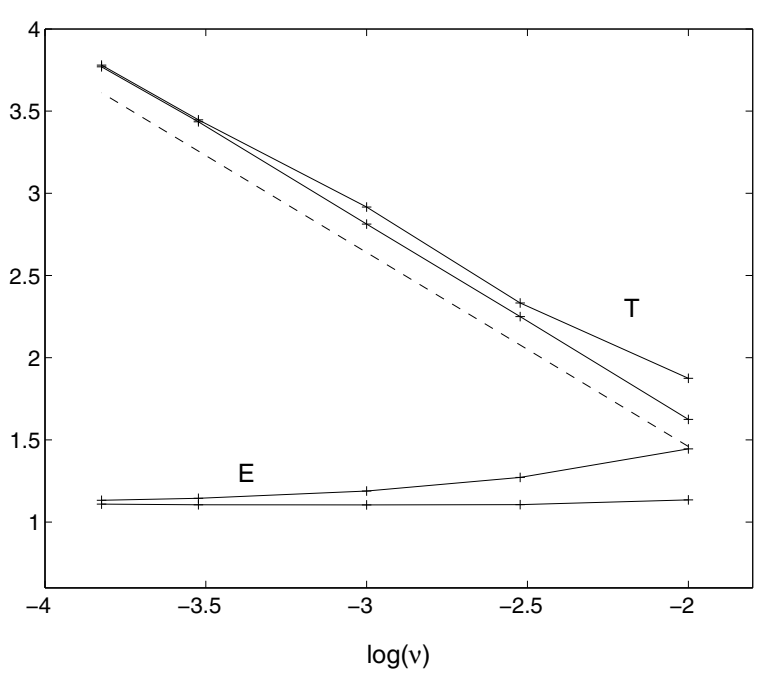

Fig. 4. Scalings of the peak energy loss rate against viscosity for the runs of Figs. 1 and 2. The solid lower lines in the sets $E$ and $T$ correspond to the classical viscosity. The marginally faster rate of the Braginskii viscosity is reflected in the slightly longer build up time $T$. The dashed reference line has a negative slope of 1.18 .

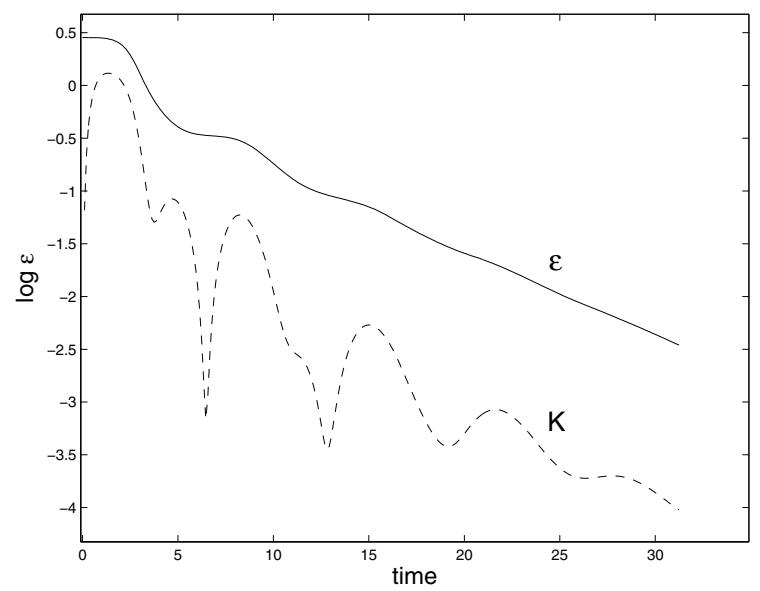

Fig. 5. Plot showing the separation of total and kinetic $(K)$ energies for a typical reconnective disturbance damped mainly by the plasma viscosity. The Braginskii viscosity is assumed with parameters $v=$ $2 \times 10^{-3}, \eta=2 \times 10^{-4}$.

a systematic increase in $T$ with reducing $v$, in accordance with the scaling $T \simeq \tau_{B} \sim \log v$, as indicated by the dashed reference line. This dependence supports the physical expectation that the size of the dissipation region scales as $r_{s} \simeq \sqrt{v}$ in all cases. On a more general level, the fact that the viscous dissipation scale is so much larger than the resistive scale $\sqrt{\eta}$, should make viscous damping a comparatively more robust energy loss mechanism.

\subsection{Reconnective planar disturbances}

In Fig. 5 we show the results of a reconnective disturbance of the form

$\psi(x, y, 0)=\left(1-x^{2}\right)\left(1-y^{2}\right)$

with $v=0$. Note that, in contrast to the previous initial condition (23), a displacement in $\psi$ has been introduced between the origin and the outer boundary that can only be eliminated by field line reconnections at the null point. The present computation assumes the Braginskii viscosity (with $v=2 \times 10^{-3}$, $\eta=0.1 v$ ) but the isotropic viscosity provides visually indistinguishable profiles, at least during the first thirty Alfvén times.

Returning to Fig. 5, we see that viscosity is extremely effective at dissipating energy during the initial implosion. But although the maximum damping rate is of order unity $(\dot{\mathcal{E}} \simeq$ $1.3, t \simeq 2.7$ ) and a dominant fraction of the disturbance energy is removed during the initial five Alfvén times, the energy loss rate begins to slow at later on. A well defined separation of the magnetic and kinetic energies has emerged for $t \geq 5$. The global energy is now almost all magnetic and declines exponentially $\mathcal{E} \sim \exp (-\gamma t)$ with $\gamma \simeq 0.18$ for the present parameters.

To interpret these results we assume that viscous damping can be strong enough to suppress the development of Alfvénic oscillatory modes, at least for reconnective disturbances (Craig et al. 2005). The system then becomes overdamped and time derivatives in the planar momentum (6) and induction (7) equations become small. Setting $\partial_{t}=0$ and eliminating the current density from (7), implies that the velocity field is controlled by the balance

$\eta \boldsymbol{F}_{\perp}=\left(\mathbf{v} \cdot \nabla \psi_{E}\right) \nabla \psi_{E}$.

Independent of which viscosity is employed, this balance requires the size scale

$r_{s}=(\eta v)^{1 / 4}$.

The emergence of a visco-resistive scale for $X$-point reconnective modes under isotropic viscosity has already been demonstrated (Craig et al. 2005), while other contexts have been provided by Park et al. (1984) and Hassam \& Lambert (1996). Our present results suggest that the visco-resistive scale also extends to the Braginskii viscosity.

Consider the Ohmic dissipation rate. We have that

$\int \eta J^{2} \mathrm{~d} V \rightarrow \eta\left(\frac{\psi}{r_{s}^{2}}\right)^{2} \pi r_{s}^{2}$.

Taking $r_{s} \simeq(\eta v)^{1 / 4}$ leads to an energy decay rate $\left.\gamma \sim \sqrt{(} \eta / v\right)$ that decreases as $v$ increases for fixed $\eta$. Note that if $\eta>v$, the viscoresistive scale is dominated by the resistive scale, so the overdamping is lost, and the oscillatory modes return. However, for $v>\eta$ with $v$ fixed, the global energy should decay as $\exp (-\gamma t)$ with $\gamma \sim \eta^{1 / 2}$ as $\eta \rightarrow 0$.

This interpretation is borne out by Fig. 6 which assumes the Braginskii viscosity for $v=10^{-2}$ and uses three values of resistivity $\left(\eta=10^{-3}, 3 \times 10^{-4}, 10^{-4}\right)$. An approximate energy scaling for these results is $\mathcal{E} \sim \exp (-\gamma t)$ where $\gamma \simeq 0.62 \sqrt{(\eta / v) \text {. The }}$ return to oscillatory behaviour when resistivity is dominant is shown in the dashed line $\left(\eta=10^{-2}, v=3 \times 10^{-3}\right)$.

\subsection{Axial field disturbances}

Axial field disturbances drive incompressive, non-reconnective motions of the plasma. When damped by the Braginskii viscosity, however, planar motions eventually develop, driven by a finite viscous force $\boldsymbol{F}_{\perp}$. An interesting question is whether these secondary motions lead to significant enhancements of the energy decay rate. This point was recently discussed by Craig \& Litvinenko (2007) who consider both linear and finite amplitude disturbances. They also point out that the highly corrugated axial field profiles that derive from phase mixing during the initial phases lead to power law energy damping $\dot{\mathcal{E}} \sim v^{1 / 3}$ for both shear and bulk viscosities (but with a reduced amplitude for the Braginskii form). In what follows we shall comment mainly on 


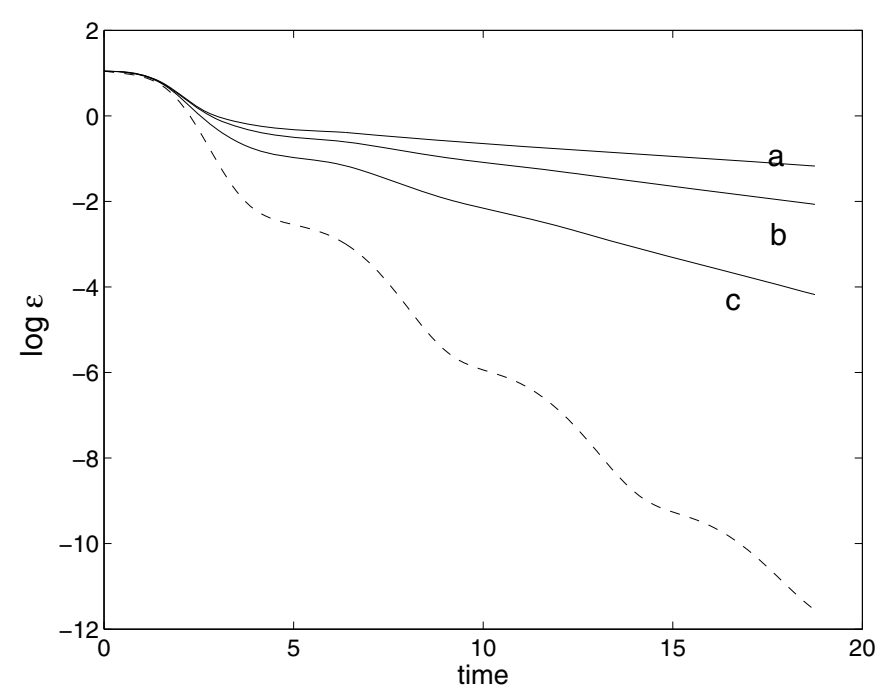

Fig. 6. Plot showing the total energy decay for the reconnective mode with Braginskii viscosity $v=10^{-2}$ for the resistivities $\eta=10^{-4}, 3 \times$ $10^{-4}, 10^{-3}$ (curves a, b, c respectively). Also shown by the dashed line is the return to oscillatory behavior in the case $\eta=10^{-2}, v=3 \times 10^{-3}$.

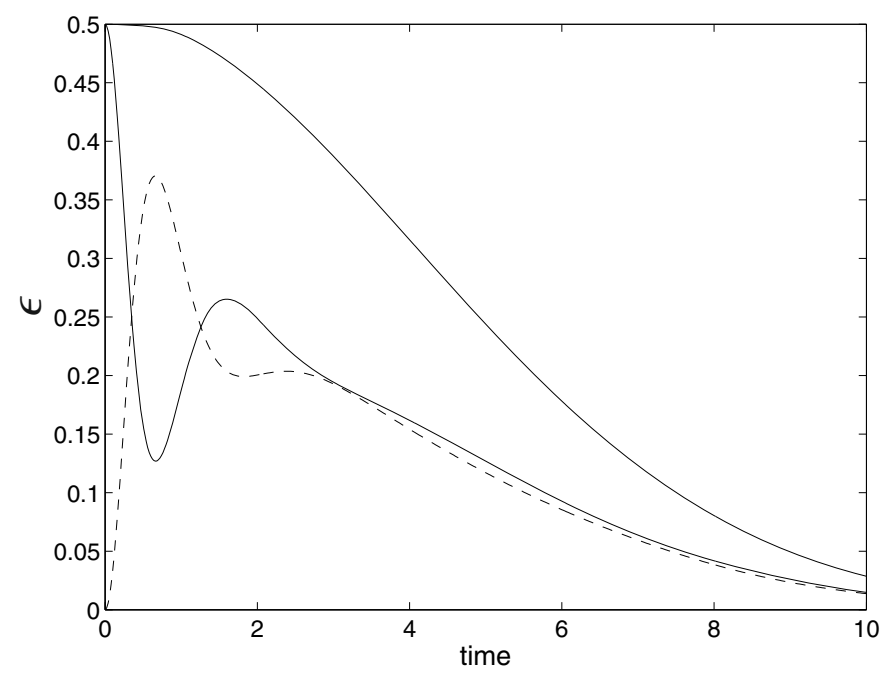

Fig. 7. Plot of total, magnetic, and kinetic (dashed line) energy versus time for axial field disturbances with $v=2 \times 10^{-3}, \eta=10^{-4}$. Note that. in marked distinction to compressible modes, the magnetic and kinetic energies decay in unison at large times

how the axial field decay compares to the decay of planar compressible disturbances.

Figure 7 shows energy decay profiles based on the initial conditions

$$
W(x, y, 0)=\sin (\pi x) \sin (\pi y), \quad Z(x, y, 0)=0,
$$

with all planar disturbances set to zero. The computation assumes a non-isotropic viscosity for the reference parameters $v=2 \times 10^{-3}$ with $\eta=0.1 v$.

It is immediately clear that energy losses, though still significant, are dramatically reduced compared to the non-reconnective planar computations of Fig. 2. For example, the peak losses in Fig. 7 are a factor of twenty down $(\dot{\mathcal{E}} \simeq 0.07, t \simeq 4)$ and occur significantly later than the corresponding losses in Fig. 2. The fact that both components of the energy decline monotonically once energy equalization has been achieved confirms that the dissipation mechanism is unrelated to the compressive

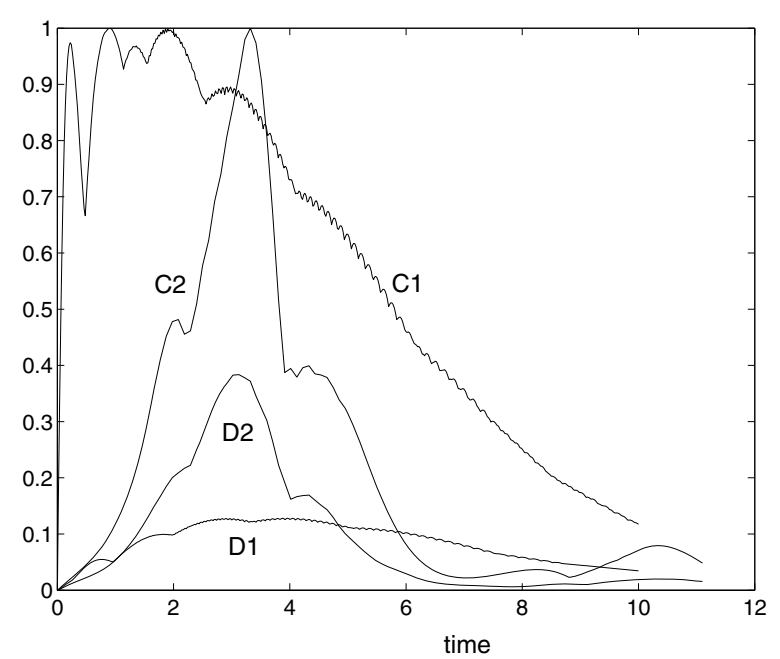

Fig. 8. Plot showing contributions to the Braginskii tensor in the case of axial (C1 and D1) and planar field disturbances (C2 and D2). Curves marked D show the contribution of the term $\nabla \cdot v$ to the scalar $S$ (29) based on the runs of Figs. 2 and 7. Compressive effects play only a minor role in the axial field computation.

oscillatory release of the planar disturbances. A further inference is that any viscous coupling between axial and planar modes can be of only minor significance. Either way, as measured by reductions in the global energy or the peak damping rate, it is clear that the Braginskii viscosity is considerably more effective for compressive disturbances.

Figure 8 compares components of the Braginskii tensor for the axial and planar computations of Figs. 2 and 7. Specifically, the curves $C 1$ and $D 1$ quantify, respectively, the individual components of the scalar

$S=\left(\frac{B_{m} B_{k}}{B^{2}} \partial_{k} v_{m}-\frac{1}{3} \nabla \cdot v\right)$,

in the case axial field disturbances. The fact that $C 1$ exceeds $D 1$ by a factor of around ten, shows that compressible effects are playing a relatively minor role in the evolution. By way of contrast, for the planar computation of Fig. 2, compressible effects are always making a substantial if not dominant contribution to the Braginskii tensor.

\section{Discussion and conclusions}

We have considered the role of anisotropic viscous damping in line-tied, coronal magnetic $X$-points. The present visco-resistive computations suggest that the Braganskii viscosity is highly effective - comparable in fact to simplified isotropic viscosity models - at damping compressive $X$-point disturbances. Notably, in the case of non-reconnective disturbances, the system behaves as an underdamped oscillator whose energy losses depend at most logarithmically on resistive or viscous dissipation coefficients. For a collisional plasma in which viscous effects dominate $(v \gg \eta)$, the bulk of the perturbation energy is released very quickly, typically within a few Alfvén times for realistic coronal parameters.

The Braginskii viscosity can also be effective in the case of reconnective disturbances. Admittedly, in the physically relevant case $v \gg \eta$, the dissipation at large times is dominated by the overdamped resistive decay of magnetic energy bound-up in the global field topology. This decay occurs very slowly, at a rate 
$\sqrt{(} \eta / v)$ that decreases (for fixed resistivity) as the level of viscous damping is increased. But the fact remains that over fifty percent of the initial disturbance energy - that which can be transferred into the kinetic energy of the fluid - is typically lost during the initial implosive collapse, well before the asymptotic phase sets in. Thus, despite the slow asymptotic rate, there can still be a rapid initial release due to the implosive localization of the reconnective disturbance.

We have also considered the influence of axial field disturbances on planar $X$-points. Generally speaking, because the Braginskii tensor suppresses cross-field viscosity components, it is not so well suited to damping incompressible shear waves that gradually steepen via phase mixing (see also Craig \& Litvinenko 2007). Simple isotropic viscosity is more effective in this regard. The Braginskii viscosity does, however, introduce a coupling between axial shearing modes and compressional planar modes that is not represented in isotropic viscous models. This coupling provides a further avenue for enhanced dissipation, but its influence on the global energy losses appears minor, at least in the case of simple $X$-point geometries.

In summary, we find that rapid visco-resistive energy release under realistic coronal conditions is most likely to be met by compressional disturbances. One difficulty is that the strong implosion required for efficient damping may be resisted by back pressures due to finite gas pressure or axial magnetic field components, effects neglected in the present treatment. For instance, in the case of purely resistive decay it is known that collapse will stall if $\beta>\eta$, a condition which is easily met in the active corona where the plasma beta is of order $10^{-2}$ (McClymont \& Craig 1996). If the damping is dominated by viscosity however, the $X$-point collapse is no longer so extreme (because $v \gg \eta$ ). Since, for a coronal plasma of several million degrees $v$ can be comparable to $\beta$, it seems likely that energy release dominated by viscous damping can be robust to the inclusion of finite gas pressure. Fast energy release by visco-resistive damping then becomes a real possibility.

Acknowledgements. This work was supported in part by the Marsden Fund (02UOW-050 MIS). Comments by Yuri Litvinenko and an anonymous referee have been appreciated.

\section{References}

Akai, A., Yoshimoto, T., Shimojo, M., \& Shibata K. 2004, ApJ, 605, L77 Birn, J., Drake, J. F., Shay, M. A., et al. 2001, J. Geophys. Res., 106, 3715 Braginskii, S. I., 1965, Rev. Plasma Phys., 1, 205

Craig, I. J. D., \& Litvinenko, Y. E. 2007, ApJ, 667, 1235

Craig, I. J. D., \& McClymont, A. N. 1991, ApJ, 371, L41

Craig, I. J. D., \& McClymont, A. N. 1993, ApJ, 405, 207

Craig, I. J. D., Litvinenko, Y. E., \& Senanayake, T. 2005, A\&A, 433, 1139

Deluca, E. E., \& Craig I. J. D. 1992, ApJ, 390, 679

Fruit, G., \& Craig, I. J. D. 2006, A\&A, 448, 753

Hassam, A. B. 1992, ApJ, 399, 159

Hassam, A. B., \& Lambert, R. P. 1996, ApJ, 472, 832

Heyverts, J., \& Priest, E. R. 1983, A\&A, 117, 220

Hollweg, J. V. 1985, J. Geophys. Res., 90, 7620

Hollweg, J. V. 1986, ApJ, 306, 730

Knoll, D. A., \& Chacon, L. 2006, Phys. Rev. Lett., 96, 135001

McClymont, A. N., \& Craig I. J. D. 1996, ApJ, 466, 487

Park, W., Monticello, D. A., \& White, R. B. 1984, Phys. Fluids, 27, 137

Priest, E. R., \& Forbes, T. 2000, Magnetic reconnection: MHD theory and applications (Cambridge Univ. Press)

Spitzer, L. 1962, Physics of fully ionized gases (John Wiley \& Sons) 